\title{
Entanglement Computation in Atoms and Molecules
}

\author{
Stefano Siccardi \\ Dip. Informatica Univ. Milano \\ via Bramante 65 \\ Crema Italy
}

\author{
Rita Pizzi \\ Dip. Informatica Univ. Milano \\ via Bramante 65 \\ Crema Italy
}

\author{
Giuliano Benenti \\ CNISM, INFN and Univ. \\ Insubria \\ via Valleggio 11 \\ Como Italy
}

\begin{abstract}
In this paper, a method for computing entanglement of electrons in atoms and molecules is described. The importance of entanglement computation for Quantum Computers and for Biology is highlighted and the existing models' pros and cons are illustrated. A description of the algorithms follows, with some considerations about the execution times and how they scale increasing the system's Hilbert space dimension.
\end{abstract}

\section{Keywords}

Quantum, entanglement, computation, algorithms.

\section{INTRODUCTION}

An intuitive way of understanding why, in the near future, quantum mechanics will become important for computation, even for classical computers, is provided by miniaturization. Smaller size circuits boost computer power because the communication between components is faster, smaller active components are faster and at the same time their density increases. The progress in miniaturization may be quantified empirically in the well-known Moore's law [1]: the number of transistors on a single integrated-circuit chip doubles approximately every $18-24$ months. Presently integrated circuits feature minimum size is about $25 \mathrm{~nm}$. If the shrinking of active elements dimensions will continue at the same rate for the next 20 years, they will probably become the same dimension of atoms and molecules. As the size of the smallest molecule, $\mathrm{H}_{2}$, is about $0.1 \mathrm{~nm}$, at that point, quantum effects will become unavoidably important. They will have in general a disturbance effect on classical computing devices, or will be used to build quantum computers. For both reasons it is important to study quantum effects in atoms and molecules, from the point of view of computing machines engineering. It is to be noted that already in the eighties Carter [2] proposed to use single molecules to build active elements of electronic circuits, but he considered only their classical behaviour. In this setting one must be sure that the quantistic behaviour does not compromise the system's functionalities. More recently the idea of quantum computers and communication systems has become feasible (see e.g. [3]); these devices will take an essential advantage from quantum effects of components that will be small enough and shielded from the environment. In this sense, it is natural to think of using single atoms or molecules to store and process both classical and quantistic information. Another point of view to motivate the study of atoms and molecules in the scope of Quantum Computers and Quantum Information is that they are well-defined systems that can be computed with high accuracy. They then constitute a firm basis to check the validity of approximated models and algorithms.

Among all the features of quantum systems, entanglement is the most relevant in quantum information. The interest in entanglement has now boomed, in sight of its possible application to Quantum Computers, Quantum Communication and quantum assisted classical Communication. Entanglement is an essential factor e.g. in quantum teleportation, quantum gates, quantum cryptography, dense coding, etc.

Many studies have dealt with many physical systems that might be good candidates for the implementation of quantum computers, and very interesting few-qubit experiments have been performed both with natural and artificial atoms and molecules, see e.g. [4], [5]. It is interesting to recall that the Nobel Prize in Physics 2012 was awarded jointly to Serge Haroche and David J. Wineland "for ground-breaking experimental methods that enable measuring and manipulation of individual quantum systems". However, even when studying natural systems, practically all the authors use some specific setting, that is well suited only to the experimental situation in which they are interested. Often these systems, if built using microelectronic techniques, are referred to as "artificial atoms" and "artificial molecules".

Moreover, a second increase of interest in entanglement has been observed, in the field of Biology, e. g. [6], [7], [8], [9]. Unfortunately, in Biology the systems are so complex that authors are often forced to resort to models borrowed from the Quantum Computers Theory. This means that phenomenological parameters are introduced, because an "ab initio" treatment is not feasible. In what follows it is proposed a way of computing the entanglement in natural atoms and molecules, that is useful both in the field of Quantum Computers and for checking the actual applicability of concepts borrowed from Quantum Information Theory to biological and biochemical systems.

\section{ENTANGLEMENT AND QUANTUM INFORMATION}

In order to make the present work as self-contained as possible, some basic concepts are now introduced. Details can be found e.g. in [3]. In Quantum Information in general one is concerned with systems defined in a finite number of states only. These states are described by a Hilbert space, that describes the system. In particular, a qubit is a system with just two states, usually denoted $|0\rangle$ and $|1\rangle$. Two or more qubits can be merged to form a bigger system, whose Hilbert space is the tensor product of the spaces of its constituents.

The state vector is an element of the Hilbert space associated with the system, e.g. $a|0>+b| 1>$ for a qubit; $a$ and $b$ are complex numbers whose squared modules are the probabilities that the system will be found in state $|0\rangle$ or $|1\rangle$ and $|\mathrm{a}|^{2}+|\mathrm{b}|^{2}=1$.

Entanglement is a property of quantum systems composed of at least two parts. A state is called entangled if it cannot be expressed by the tensor product of states of the subsystems; in this situation it is not possible to assign them individual state vectors and intriguing non-classical properties arise (see [3]). 
To find the stationary state vectors $|\Psi\rangle$ of a system one has to solve the Schroedinger equation for the system at hand, that is the equation

\section{$\mathrm{H} \Psi=\mathrm{E} \Psi$}

where $\mathrm{H}$ is the Hamiltonian and E, the Hamiltonian's eigenvalues, represent the possible energy values of the system.

In particular, the Hamiltonian of the He atom has the form:

$\mathrm{H}=\mathrm{K}+\mathrm{N}+\mathrm{I}+\mathrm{S}$

where $\mathrm{K}$ is the kinetic energy of the two electrons, $\mathrm{N}$ represents the attraction by the nucleus (inversely proportional to the distances $r_{1}, r_{2}$ of the two electrons from the nucleus), I the electron-electron repulsion (inversely proportional to their mutual distance, $\mathrm{r}_{12}$ ) and $\mathrm{S}$ contains spin dependent terms. A similar equation holds for molecules in the BornOppenheimer approximation (see [10] and [11]).

It is also important to recall from atomic physics that the electrons can be located in several shells, more or less far from the nucleus, labeled with letters: S, P, D, F etc. Moreover, neglecting the spin-orbit interactions, the state vector factorizes into two terms, one related to the spins and the other to the orbits of the electrons. The orbital state vector, the only one that will be considered in the present work, must be symmetric (for states called singlets) or antisymmetric (for states called triplets), with respect to exchanging the two electrons. The terms singlet and triplet refer to configurations of the spin degrees of freedom (see [10] and [11]).

Standard high precision results about energies were used to check that the results of the method proposed in the present paper did not substantially differ from more standard descriptions of the atom.

The density matrix is an alternative way to completely describe a quantum system. For the following discussion, the density matrix is obtained by

$\rho=|\Psi\rangle\langle\Psi|$

A reduced density matrix of a system composed of several parts is obtained computing a partial trace of the density matrix of the total system on one of its subsystems.

The von Neumann entropy of a system described by a density matrix $\rho$ is defined by

$S(\rho)=-\operatorname{Tr}(\rho \log \rho)$

and can be computed using the eigenvalues $\lambda_{\mathrm{i}}$ of $\rho$ as

$S(\rho)=-\Sigma_{i} \lambda_{i} \log \lambda_{i}$

It is the analogous of the classical Shannon entropy for quantum information. The von Neumann entropy of the reduced matrix of a bipartite system is often used to quantify its entanglement; when it is not possible to compute the von Neumann entropy, the linear entropy is used, $S_{L}=1-\operatorname{Tr}\left(\rho^{2}\right)$. It is to be noted that the entanglement definition, in this case for the Helium electrons, be it based on the von Neumann or on the linear entropy, is still debated and may differ according to several authors. The reader is referred to the discussion in [12].

\section{THE STATE OF THE ART}

For implementation of Quantum Computers several systems have been studied.
For instance [13] reviewed the research about entangled states of trapped atomic ions. In Quantum Information processing applications, a group of atomic ions is confined in a particular arrangement of electric fields. In this way one gets a collection of quantum systems that can be individually manipulated, their states entangled, and their coherences maintained for long time. Two specific internal states of each ion are selected, and entanglement between pairs of ions is studied. [14] considered neutral atoms in optical lattices. Ultracold atoms are loaded into three-dimensional arrays of microscopic trapping potentials, known as optical lattices, in such a way that every lattice site is occupied by a single atom. After initialization, the interactions and the states of the atoms are controlled to coax them into the correct entangled state.

Rydberg atoms, that is atoms with an external electron in a highly excited state, have been extensively used in quantum information experiments, as reviewed in [15]. Also in these cases, in general, two specific levels are selected, and the experiments involve the interactions and entanglement of Rydberg atoms and photons or of couples of Rydberg atoms.

Also polar molecules have been considered to realize qubits, see e.g. [16]. The basic building block is a system of two polar molecules strongly trapped at given sites of an optical lattice, where the qubit is represented by a single electron outside a closed shell of a molecule formed by more than one element in its rotational ground state.

All the above cases share the fact that 1) only a few states of the systems are selected, often just the two levels that are necessary to engineer a qubit, and the experimental setting tends to exclude all the other states; 2) the systems are artificially kept in the experimental environment. So, however interesting, they do not study entanglement in natural conditions.

On the other hand, studying natural atoms and molecules in their natural conditions, as proposed in the present work, has two important advantages as it can shed light on: 1) the difficulties that one will meet when the dimensions of the components of a classical computer will become of the order of magnitude of molecules and atoms, and natural quantum effects in the materials will interfere with the computer operations 2) the possibility of using natural molecules, or polymers, to hold active elements (gates, etc.). In this way one could achieve the maximal miniaturization, probably the same of biological systems.

Helium is the simplest case of a two electron system. Studying the entanglement in this system one obtains reference values to compare with the results of the simplified methods that must be employed when dealing with more complex systems.

In Biology several phenomenological models have been proposed. There is a family of models used to model active sites, see for instance [8] and [17]. The physical elements that are relevant for the phenomenon of interest, e.g. the "chromophores" in photosynthesis, are described as two level systems (qubits), that can be grouped in higher level structures. A Hamiltonian function is then derived for these structures, using measures of site energies and coupling strengths. In general, a term is added to take into account the interplay between internal coherent dynamics and decoherence effects due to environmental interactions.

The term describing the dynamics of the $\mathrm{N}$ active elements considered as closed systems, is usually written in the form: 
$\mathrm{H}_{\text {int }}=\Sigma \mathrm{E}_{\mathrm{i}} \mid \mathrm{i}>\langle\mathrm{i}|+\Sigma_{\mathrm{i}} \Sigma_{\mathrm{j}>\mathrm{i}} \mathrm{J}_{\mathrm{ij}}(\mid \mathrm{i}>\langle\mathrm{j}|+| \mathrm{j}>\langle\mathrm{i}|)$

here $\mid i>$ represents the state in which only the ith element is excited, $E_{i}$ are the on-site energies and $\mathrm{J}_{\mathrm{ij}}$ are the coupling strengths, both measured experimentally.

Some realistic hypotheses on the environmental properties are added, then numerical values for the relevant quantities are derived from the experiments and used in simulations to evaluate the entanglement.

For the purposes of the present work, it is important to stress that, however detailed, these models do not start from the basic chemical structures involved. The approach that is herein proposed consists, on the contrary, to start from the bottom level of intra atomic entanglement, with the goal of eventually arriving at the level of the phenomenological description. In this way, models like those described above will be completely justified.

Some simplified models have been proposed. E.g. [18], [19], [20], [21], [22], [23], used analytical models to compute the entanglement in atoms. Although these papers highlight some interesting aspects of the problem, it must be stressed that all of them use some simplifications in the Hamiltonian, so that the problem can be treated analytically. Instead of the complete Hamiltonian, these models use Hamiltonians like the following:

$\mathrm{H}=\mathrm{K}+\mathrm{A}+\mathrm{P}$

Where $\mathrm{A}$ is a harmonic potential and $\mathrm{P}$ is the interaction potential, taken proportional to $\mathrm{r}_{12}^{2}$ or to $1 / \mathrm{r}^{2}{ }_{12}$ or to $1 / \mathrm{r}_{12}$. As a consequence, none of those models can be considered a high precision description of a real atom.

In two papers, [24], [25], the authors computed the von Neumann entropy of a two electron system, a heliumlike atom, near the ionization threshold. Their model considers again a simplified Hamiltonian, namely one of the form:

$\mathrm{H}=\mathrm{K}+\mathrm{N}+\lambda / \mathrm{r}$,

where $\mathrm{N}$ represents the attraction by the nucleus (inversely proportional to the nucleus-electron distance $)$ and $r_{>}=\max \left(r_{1}\right.$; $r_{2}$ ). This means that the models uses a spherical average of the repulsion between electrons. In [24] the von Neumann entropy of the ground state only of Helium is computed, focusing on its dependence on the "coupling" parameter $\lambda$ and on the search of its critical values. In [25] a similar model is used for the lowest energy triplet state.

A computation of entanglement for a realistic model of the Helium atom can be found in [26]. The entanglement is evaluated computing the linear entropy, that implies to evaluate the trace of the squared reduced density matrix. As the functions used as a basis in this work are expressed in terms of spatial coordinates, no simplifications are possible and it is necessary to evaluate a 12-dimensional integral, that was computed using Monte Carlo multidimensional numerical integration. The authors computed the linear entropy for several levels of the singlet and triplet; actually, the present work can be considered an extension of [26], as some more levels have been computed, and also the von Neumann entropy has been evaluated, because the method proposed herein computes the eigenvalues of the reduced density matrix.

Another difference is that the Monte Carlo integration implies statistical errors. On the contrary, in the proposed method, once one has attained a requested precision in the energy, the entropies are computed using only algebraic operations, so no other approximations or errors are involved.

A comparison between existing techinques is summarized in Table 1; the main differences considered are: "cond", the conditions of the systems (Artificial, Simplified or Natural); "ab initio" computations (Yes, No or Not applicable); "energy approximation" (High, Good, Low or Not applicable); "vN entr" if the model is able to compute the von Neumann entropy (Yes, Approximated, No); "lin entr" the same for linear entropy.

Results obtained for Helium have been reported in [12].

Table 1. Comparison between existing techiques and the proposed scheme

\begin{tabular}{|c|c|c|c|c|c|}
\hline model & cond & $\begin{array}{c}\text { ab } \\
\text { initio }\end{array}$ & $\begin{array}{c}\text { energy } \\
\text { appr }\end{array}$ & $\begin{array}{c}\text { vN } \\
\text { entr }\end{array}$ & $\begin{array}{c}\text { lin } \\
\text { entr }\end{array}$ \\
\hline $\begin{array}{c}\text { For QC } \\
{[13][14]} \\
{[15][16]}\end{array}$ & Art & Yes & High & Yes & Yes \\
\hline $\begin{array}{c}\text { For } \\
\text { Biology } \\
{[8][17]}\end{array}$ & Simp & No & Not app & Appr & Appr \\
\hline $\begin{array}{c}\text { Analytical } \\
\text { models } \\
{[18][19]} \\
{[20][21]} \\
{[22][23]}\end{array}$ & Simp & Not & Low & Appr & Appr \\
\hline $\begin{array}{c}\text { Near } \\
\text { ionization } \\
{[24][25]}\end{array}$ & Simp & Yes & Good & Appr & Appr \\
\hline $\begin{array}{c}\text { Helium } \\
\text { as in } \\
{[26]}\end{array}$ & Nat & Yes & High & No & Appr \\
\hline $\begin{array}{c}\text { Present } \\
\text { method } \\
{[12]}\end{array}$ & Nat & Yes & Good & Yes & Yes \\
\hline
\end{tabular}

\section{DESCRIPTION OF THE ALGORITHM}

The computation is composed of the following steps:

Step 1: computation of the energy of a specific state of the system. The Schroedinger equation is solved using the variational method. It consists of defining solutions that depend on variational parameters. This can be considered as a parametrization of the Hilbert space. The method is based on a theorem, stating that upper bounds of energies can be found looking for minima of the parameters, both for the fundamental and the excited states. The estimate of energies is improved with a suitable choice of the initial wave functions. There are 3 substeps:

Step 1.1: computation of the matrix element of the Hamiltonian $\mathrm{H}$ of the system

Step 1.2: computation of eigenvalues and eigenvectors of this matrix

Step 1.3: evaluation of the minimum of the eigenvalues, varying the parameters

Step 2: computation of entanglement, with the substeps

Step 2.1: computation of the density matrix and reduced density matrix using the eigenvectors computed in step 1

Step 2.2: computation of the eigenvalues of the reduced density matrix, then computation of the entropies. 
Step 2.3: computation of entanglement given the entropy. As already noted, entanglement can be defined in several ways (see [12]).

Step 1.1. Details for Helium: an orthonormal basis is used for the Hilbert space, with functions:

$$
\Phi_{\mathrm{n} 1,11, \mathrm{~m} 1 ; \mathrm{n} 2,12, \mathrm{~m} 2}=\mathrm{F}_{\mathrm{n} 1,11 ; \mathrm{n} 2,12}\left(\mathrm{r}_{1}, \mathrm{r}_{2}\right) \mathrm{Y}_{11 \mathrm{~m} 1}\left(\Omega_{1}\right) \mathrm{Y}_{12 \mathrm{~m} 2}\left(\Omega_{2}\right)
$$

where the indexes 1,2 refer to the two electrons, the $\mathrm{Y}$ are spherical harmonics, that depend on the atomic shell considered and the $\Omega$ are the solid angles for each particle, $1_{1}$, $l_{2}=0,1,2$ for shells $S, P, D$. The radial functions $F$ are obtained starting with the functions

$\mathrm{R}_{\mathrm{nl}}(\mathrm{r})=\mathrm{r}^{\mathrm{n}+\mathrm{l}-1} \exp \left(-\xi_{\mathrm{n}, \mathrm{l}} \mathrm{r}\right)$

known as Slater Type Orbitals (STO) in atomic physics. Here $\xi_{\mathrm{n}, \mathrm{l}}$ are variational parameters. These $\mathrm{R}$ functions are orthonormalized obtaining a set of functions $f_{n l}(r)$. The $F$ functions are then defined as:

$$
F_{n 1,11 ; n 2,12}\left(r_{1}, r_{2}\right)=(1 / 2)^{1 / 2}\left(f_{n 111}\left(r_{1}\right) f_{n 212}\left(r_{2}\right)-f_{n 212}\left(r_{1}\right) f_{n 111}\left(r_{2}\right)\right)
$$

for the antisymmetric case and

$$
F_{n 1,11 ; n 2,12}\left(r_{1}, r_{2}\right)=(1 / 2)^{1 / 2}\left(f_{n 111}\left(r_{1}\right) f_{n 212}\left(r_{2}\right)+f_{n 212}\left(r_{1}\right) f_{n 111}\left(r_{2}\right)\right)
$$

or $F_{n 1,11: n 1,11}\left(r_{1}, r_{2}\right)=f_{n 111}\left(r_{1}\right) f_{n 111}\left(r_{2}\right)$ for the symmetric. Up to 3 shells ( $\mathrm{S}, \mathrm{P}$ and $\mathrm{D})$ have been considered in our computations and after several tests it was concluded that it is sufficient to consider a single variational parameter $\xi$ for each shell. The Hamiltonian matrix elements are computed using the functions $\mathrm{F}$, that means to compute the kinetic energy and the potential energies for the nucleus attraction and mutual repulsion of the electrons, integrating on the whole space.

Details for $\mathbf{H}_{2}$ : in this case there is the further complication that there are two nuclei at some distance R. As usual, to compute energies and electronic eigenfunctions the BornOppenheimer approximation has been used, that considers nuclei at a fixed distance. For this reason it is convenient to approximate every STO function with a suitable number of Gaussian functions. The great advantage of the Gaussian representation, based on the linear combination of Gaussian functions, is that for instance an integral over a product of two Gaussians centered about two positions reduces to a single integral over a third Gaussian centered in an intermediate point; similar results are available for more complex cases ([27]).

Step 1.2 In all the runs the matrix to diagonalize had dimension up to $250 \times 250$. The computation of eigenvalues and eigenvectors of a matrix is a well-studied topic, see e.g. [28] where the calculation of electronic structure is quoted as a classical eigenvalue problem. Any algorithms can be used (classical Jacobi, divide and conqueror, parallelized algorithms, etc.) to find the best mix of simplicity, speed, and accuracy. It is to be noted that the $\mathrm{H}$ matrix is symmetric.

Step 1.3 It was found that a single variational parameter for each shell is sufficient, and that the minimization can be performed one shell at a time, so that simple methods for finding local minima of functions of one variable can be employed.

Step 2.1 The reduced density matrix is obtained integrating

f $\Psi\left(\mathbf{r}_{1}, \mathbf{r}_{2}\right) \Psi *\left(\mathbf{r}_{1}{ }_{1}, \mathbf{r}_{2}\right) \mathrm{d} \mathbf{r}_{2}$ where the $\Psi$ have been computed in Step 1 in terms of the $\Phi$ functions computed in Step 1.1:

$\Psi\left(\mathbf{r}_{1}, \mathbf{r}_{2}\right)=\Sigma_{\mathrm{i}, \mathrm{k}} \mathrm{c}_{\mathrm{i}, \mathrm{k}} \Phi_{\mathrm{i}, \mathrm{k}}\left(\mathbf{r}_{1}, \mathbf{r}_{2}\right)$, here multi-indexes i,k have been used for the sake of brevity. Since the expansion is done over an orthonormal basis the reduced density matrix on that basis is simply given by a partial trace over the second particle of the overall density matrix. Schematically:

$\rho^{\text {rid }}{ }_{i, k}=\Sigma_{j} c_{i, j} c_{k, j}$

A major advantage of the method and the use of orthonormal basis orbitals is that the reduced density matrix is obtained by purely algebraic methods, without numerical computations of multi-dimensional integrals.

Step 2.2 It consists again of an eigenvalue problem, but with two simplifications: 1) the matrices are smaller 2) eigenvectors are not needed. Once eigenvalues are found, every form of entropy that is needed can be computed.

\section{PERFORMANCE EVALUATIONS}

In order to evaluate critical points and to test the possibility to use the method in more complex situations, the time needed to perform the main subroutines of the Fortran implementation was measured. All the calls in a program run have been summed to compute tables 2, 3, 4 and 5. The entries $=0$ mean that the time was negligible. In all the tables, $\mathrm{n}$ is the dimension of the single particle Hilbert space. The total run time for $n=3$ has been taken as a unit of measure: the figures are relative measures, exploiting the time scaling with $\mathrm{n}$.

Here eig1 computes the eigenvalues of the reduced density matrix; it scales linearly with $\mathrm{n}$ in the range considered; eig2 computes the eigenvalues of the Hamiltonian. For two particles the dimension is $n(n+1) / 2$ for singlets and $n(n-1) / 2$ for triplets; eig2 scales approximately as $\mathrm{n}^{5.8}$. ortho orthogonalizes the STO functions, and scales linearly. The routine singp computes the matrix elements for a single electron, and twop for two electrons in the same shell; they scale respectively as $\mathrm{n}^{4.6}$ and $\mathrm{n}^{3.5}$. The total runtime, for $\mathrm{n}>7$ scales as $n^{3.8}$. The programs have been compiled with the gfortran compiler and run on two Windows computers; and with the Intel compiler on a Linux system. Average run times have been computed to compare the subroutines scaling.

Table 2. Execution time - He S shell only

\begin{tabular}{|c|c|c|c|c|c|c|}
\hline $\mathbf{n}$ & eig1 & eig2 & Ortho & singp & twop & Total \\
\hline 3 & 0.0000 & 0.0003 & 0.0000 & 0.0023 & 0.0010 & 1 \\
\hline 5 & 0.0013 & 0.0043 & 0.0003 & 0.0144 & 0.0020 & 1.0187 \\
\hline 7 & 0.0030 & 0.0314 & 0.0007 & 0.0725 & 0.0047 & 1.1089 \\
\hline 9 & 0.0070 & 0.1416 & 0.0013 & 0.2438 & 0.0124 & 1.4021 \\
\hline 11 & 0.0150 & 0.4693 & 0.0030 & 0.6536 & 0.0294 & 2.1690 \\
\hline 13 & 0.0210 & 1.3133 & 0.0040 & 1.4957 & 0.0655 & 3.9092 \\
\hline 15 & 0.0361 & 2.9312 & 0.0073 & 3.0361 & 0.1346 & 7.1523 \\
\hline 17 & 0.0558 & 6.0564 & 0.0104 & 5.6673 & 0.2672 & 13.063 \\
\hline 19 & 0.0815 & 12.123 & 0.0147 & 9.7993 & 0.4873 & 23.516 \\
\hline
\end{tabular}

Table 3. Execution time - He S and P shells

\begin{tabular}{|c|c|c|c|c|c|c|c|}
\hline $\mathbf{n}$ & eig1 & eig2 & $\begin{array}{c}\text { Orth } \\
\mathbf{0}\end{array}$ & $\begin{array}{c}\text { sing } \\
\mathbf{p}\end{array}$ & twop & twoq & $\begin{array}{c}\text { Tota } \\
\mathbf{l}\end{array}$ \\
\hline 5 & $\begin{array}{c}0.00 \\
0\end{array}$ & $\begin{array}{c}0.04 \\
0\end{array}$ & 0.001 & $\begin{array}{c}0.02 \\
7\end{array}$ & $\begin{array}{c}0.00 \\
5\end{array}$ & $\begin{array}{c}0,00 \\
4\end{array}$ & $\begin{array}{c}1.00 \\
0\end{array}$ \\
\hline 7 & $\begin{array}{c}0.00 \\
1\end{array}$ & $\begin{array}{c}0.28 \\
8\end{array}$ & 0.001 & $\begin{array}{c}0.13 \\
6\end{array}$ & $\begin{array}{c}0.01 \\
1\end{array}$ & $\begin{array}{c}0.00 \\
8\end{array}$ & $\begin{array}{c}1.36 \\
1\end{array}$ \\
\hline 9 & $\begin{array}{c}0.00 \\
2\end{array}$ & $\begin{array}{c}1.27 \\
7\end{array}$ & 0.002 & $\begin{array}{c}0.45 \\
9\end{array}$ & $\begin{array}{c}0.02 \\
8\end{array}$ & $\begin{array}{c}0.01 \\
8\end{array}$ & $\begin{array}{c}2.70 \\
1\end{array}$ \\
\hline
\end{tabular}




\begin{tabular}{|c|c|c|c|c|c|c|c|}
\hline 1 & 0.00 & 4.47 & \multirow{2}{*}{0.005} & 1.21 & 0.06 & 0.04 & 6.70 \\
\hline 1 & 3 & 1 & & 0 & 8 & 2 & 1 \\
\hline 1 & 0.00 & 11.8 & \multirow{2}{*}{0.008} & 2.77 & 0.14 & 0.09 & 15.8 \\
\hline 3 & 5 & 5 & & 5 & 9 & 0 & 0 \\
\hline 1 & 0.00 & 27.1 & \multirow{2}{*}{0.012} & 5.64 & 0.29 & 0.17 & 34.2 \\
\hline 5 & 8 & 6 & & 5 & 9 & 5 & 2 \\
\hline
\end{tabular}

Table 3 refers to runs in which electrons in shell $\mathrm{S}$ and $\mathrm{P}$ have been considered. The name of the routines is the same as in table 2 with the exception of twoq that computes the interactions between electron in different shells. The scaling of this routine is $n^{3.5}$, all the others are similar to the case of shell S only.
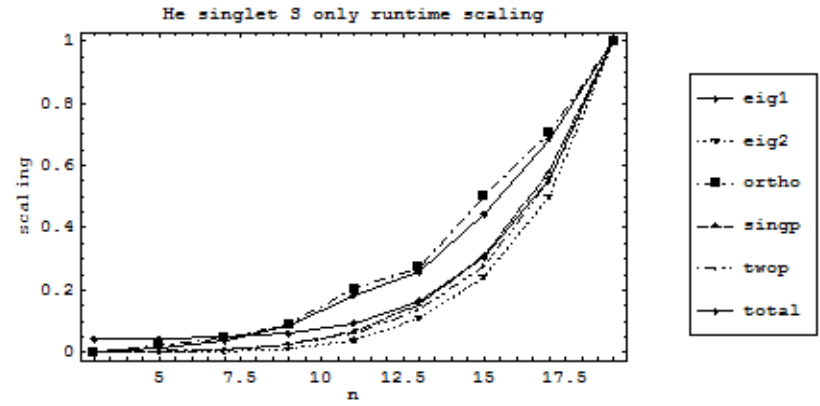

Fig 1: The scaling routines for shell $S$
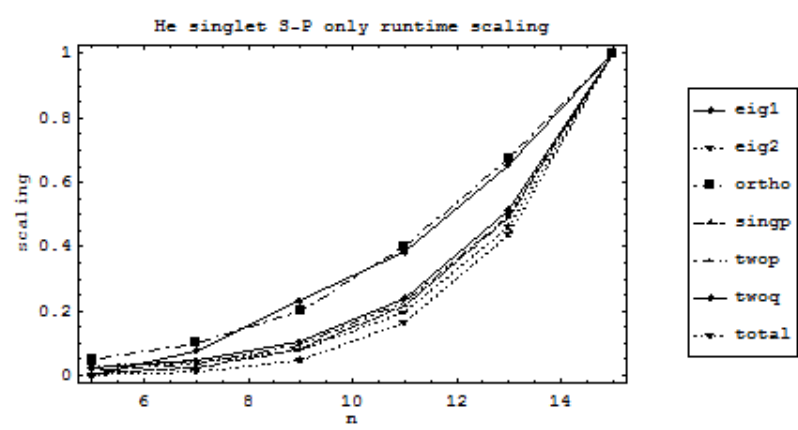

Fig 2: The scaling routines for shell $\mathbf{S}$ - $\mathbf{P}$

Figures 1 and 2 represent graphically the scaling of the subroutines, relative to the longest time of each of them.

Also the total run times for runs considering only the S, the S$\mathrm{P}$ and the S-P-D shells have been compared. Mean times in seconds are shown in table 4. For these runs we used a 1.66 Ghz, $1 \mathrm{~Gb}$ Ram, 32 bit Windows system. The ratio between the $\mathrm{S}$ and $\mathrm{S}-\mathrm{P}$ runs scales approximately as $\mathrm{n}^{1.5}$, and the ratio between $S$ and S-P-D runs as $n^{2.6}$.

Table 4. Execution time - He S, S-P, S-P-D shells

\begin{tabular}{|c|c|c|c|c|c|}
\hline $\mathbf{n}$ & $\mathbf{S}$ & $\mathbf{S - P}$ & S-P-D & S-P / S & S-P-D / S \\
\hline 5 & 47.6 & 50.6 & 56.8 & 1.06 & 1.19 \\
\hline 7 & 51.8 & 68.8 & 115.3 & 1.33 & 2.23 \\
\hline 9 & 65.5 & 136.5 & 350.3 & 2.08 & 5.35 \\
\hline 11 & 101.3 & 339.4 & 1032.5 & 3.35 & 10.19 \\
\hline 13 & 182.6 & 798.4 & 2598.6 & 4.37 & 14.23 \\
\hline 15 & 334.1 & 1728.9 & 6525.2 & 5.18 & 18.72 \\
\hline
\end{tabular}

For the $\mathrm{H}_{2}$ computation, the scaling with the number of basis functions and with the number $\mathrm{G}$ of Gaussian functions used in the expansions has been considered. Table 5 shows that, notwithstanding some fluctuations due to the small values of $n$ used in these exploratory runs, the time needed to compute the two bodies interactions is far longer than the duration of all the other subroutines. Times are relative to the total run time for $n=3, G=1$.

Table 5. Execution time $-\mathrm{H}_{2} \mathrm{~S}$ shell only

\begin{tabular}{|c|c|c|c|c|c|c|}
\hline $\mathbf{n}$ & G & eig1 & eig2 & ortho & twop & Total \\
\hline 3 & 1 & 0.0000 & 0.0294 & 0.0009 & 0.0069 & 1 \\
\hline 3 & 3 & 0.0000 & 0.0025 & 0.0057 & 0.3173 & 1.288 \\
\hline 3 & 6 & 0.0000 & 0.0018 & 0.0261 & 4.4026 & 5.393 \\
\hline 5 & 3 & 0.0003 & 2.0691 & 0.0186 & 2.8533 & 5.905 \\
\hline 5 & 6 & 0.0006 & 0.1536 & 0.0742 & 40.6721 & 41.863 \\
\hline
\end{tabular}

\section{CONCLUSIONS}

An algorithm to compute electronic entanglement in atoms and molecules has been described. All tests showed that the entanglement computation is feasible with less effort than energy computation, and that the algorithm can be extended, without major problems, to more complex situations

Moreover, these computations that are in principle "exact" as their precision is limited only by the Hilbert space dimension, allow to check the accuracy of simplified computational models that must be used in situations that are so complex that approximate methods are mandatory. Among these, there are undoubtedly biochemical and biological systems, where the entanglement has been proposed as an important efficiency factor.

Other applications can be found in the quantum computer implementation area, where natural atoms and molecules could be considered as candidates to realize active elements.

Even classical computers could benefit of this kind of computation, as quantum effects will show with the progress of miniaturization, and it will be important to prevent their interference with the optimal operation of the devices.

\section{REFERENCES}

[1] G.E. Moore (1965); Cramming more components onto integrated circuits Electronics, 38, N. 8

[2] F.L. Carter (1987); Molecular electronic devices, Dekker Inc.

[3] G. Benenti, G. Casati, G. Strini (2007); Principles of quantum computation and information, vol. I-II, World Scientific

[4] G. Benenti, G. Strini (2007); A bird's eye view of quantum computers arXiv:quant-ph/0703105, Quantum Biosystems 1, 21

[5] I. Buluta, S. Ashhab, F. Nori (2011); Natural and artificial atoms for quantum computation Reports on Progress in Physics, 74, p. 104401

[6] G. S. Engel, T.R. Calhoun, E. L. Read1 T-K. Ahn, T. Manal, Y-C. Cheng, R. E. Blankenship, G. R. Fleming (2007); Evidence for wavelike energy transfer through quantum coherence in photosynthetic systems Nature, 446 , p.782

[7] M. Mohseni, P. Rebentrost, S. Lloyd, A. Aspuru-Guzik (2008); Environment-assisted quantum walks in photosynthetic energy transfer Journal of Chemical Physics, 129, p.174106 
[8] M. Sarovar, A. Ishizaki, G. R. Fleming, and K. B. Whaley (2010); Quantum entanglement in photosynthetic light harvesting complexes Nature Physics, 6, 462 (2010).

[9] T. Scholak, F. de Melo, T. Wellens, F. Mintert1, A. Buchleitner (2011); Efficient and coherent excitation transfer across disordered molecular networks Physical Review E, 83, p. 021912

[10] M. Weissbluth (1978), Atoms and molecules, Academic Press

[11] J. D. Graybeal (1993); Molecular spectroscopy, McGraw-Hill

[12] G. Benenti, S.Siccardi, G. Strini (2012); Entanglement in Helium arXiv:quant-ph/1204.6667

[13] R. Blatt, D. Wineland (2008); Entangled states of trapped atomic ions Nature, 453, p.1008

[14] I. Bloch (2008); Quantum coherence and entanglement with ultracold atoms in optical lattices Nature, 453, p. 1016

[15] M. Saffman, T.G. Walker, K. Molmer (2010); Quantum information with Rydberg atoms Reviews of Modern Physics 82, p.2313

[16] A. Micheli, G.K. Brennen, P. Zoller (2006); A toolbox for latticespin models with polar molecules Nature Physics 2, p.341

[17] K. B. Whaley, M. Sarovar, A Ishizaki (2011); Quantum entanglement phenomena in photosynthetic light harvesting complexes, Procedia Chemistry, 3, p. 152

[18] C. Amovilli, N. H. March (2003); Exact density matrix for a two-electron model atom and approximate proposals for realistic two-electron systems Phisical Review A, 67, p. 22509
[19] C. Amovilli, N. H. March (2004); Quantum information: Jaynes and Shannon entropies in a two-electron entangled artificial atom Physical Review A, 69, p. 54302

[20] M. Moshinsky (1968) How good is the Hartree-Fock approximation? The American Journal of Physics, 36, p. 52

[21] A. Nagy (2006); Fisher information in a two-electron entangled artificial atom Chemical Physics Letters, 425, p. 154

[22] N. H. March, A. Cabo, F. Claro, G. G. N. Angilella (2008) Proposed definitions of the correlation energy density from a Hartree-Fock starting point: the twoelectron Moshinsky model atom as an exactly solvable model Physical Review A, 77, p. 042504 (2008)

[23] R. J. Yanez, A. R. Plastino, J. S. Dehesa (2010) Quantum entanglement in a soluble two-electron model atom European Physics Journal D, 56, p. 14

[24] O. Osenda, P. Serra (2007) Scaling of the von Neumann entropy in a two-electron system near the ionization threshold Physical Review A, 75, p. 042331

[25] O. Osenda, P. Serra (2008) Excited state entanglement on a two-electron system near the ionization threshold Journal of Physics B, 41, p. 065502

[26] J. S. Dehesa, T. Koga, R. J. Yanez, A. R. Plastino, and R. O. Esquivel (2012); Quantum entanglement in helium Journal of Physics B 45, p. 015504

[27] R. Daudel, G. Leroy, D. Peters, M. Sana, (1983) Quantum Chemistry, Wiley

[28] Y. Saad (2011); Numerical methods for large eigenvalue problems SIAM Philadelphia 2011 